\title{
Laparoscopic versus laparotomy approach to endometrial cancer: A prospective study
}

\author{
López-De la Manzanara Carlos ${ }^{1}$, Martín-Francisco Cástor ${ }^{1}$, Luengo-Tabernero Ángel ${ }^{1}$, \\ Garrido-Esteban Rosa Ana ${ }^{1^{*},}$ León-Martín Alberto ${ }^{2}$, Haya-Palazuelos Javier ${ }^{1}$ \\ ${ }^{1}$ Department of Obstetrics \& Gynecology, Ciudad Real University General Hospital, Ciudad Real, Spain \\ ${ }^{2}$ Research Department, Ciudad Real University General Hospital, Ciudad Real, Spain \\ Email: ${ }^{*}$ clmanzanara@hotmail.com
}

Received 19 April 2013; revised 17 May 2013; accepted 23 May 2013

Copyright (C) 2013 López-De la Manzanara Carlos et al. This is an open access article distributed under the Creative Commons Attribution License, which permits unrestricted use, distribution, and reproduction in any medium, provided the original work is properly cited.

\begin{abstract}
Objective: The aim of this study was to compare laparoscopic (LPS) and laparotomy (LPT) approaches for endometrial cancer, and to assess intraoperative and postoperative results, disease-free survival and overall survival. Methods: We designed a prospective observational study, every patient diagnosed of endometrial cancer and subsidiary to surgical staging was included. Total hysterectomy, bilateral adnexectomy, pelvic lymphadenectomy were performed in every case. Paraaortic lymphadenectomy was performed depending on tumor histology. Results: 70 patients with endometrial cancer were enrolled, 49 $(70 \%)$ were treated laparoscopically and $21(30 \%)$ laparotomically. There was not statistical significant difference in the mean operative time, it was $183.06 \pm$ 21.03 min (range 120 - 230) in the LPS group and $195.24 \pm 28.39$ min (range $130-240$ ) in the LPT group, mean difference 12.16 (95\% CI -0.2 - 24). There was no difference in the number of lymph nodes resected. Mean blood loss was lower in the LPS group. There were less postoperative complications, 3 $(6.12 \%)$ in the LPS group and $7(33.3 \%)$ in the LPT group $(\mathbf{p}<0.01)$. Mean hospital stay was significantly shorter in the LPS group $4.29 \pm 1.62$ days vs $8.81 \pm$ 3.37 days in the LPT group $(p<0.01)$, mean difference 4.52 (95\% CI 3.3 - 5.7). Overall survival was similar in both groups. Conclusion: Laparoscopic approach for endometrial cancer offers similar results in terms of survival and oncological radicality as the laparotomic approach and a lower rate of complications, a quicker convalescence time and a shorter hospital stay.
\end{abstract}

\footnotetext{
Corresponding author.
}

Keywords: Endometrial Cancer; Laparoscopy; Laparotomy; Minimally Invasive Surgery; Survival

\section{INTRODUCTION}

Endometrial cancer is the most common gynecologic malignancy in the developed countries. Annually there are approximately 47,100 new cases and 8000 deaths from the disease in the USA [1]. Incidence rates of endometrial cancer have been raising due to increasing life expectancy as this disease frequently appears in women older than 55 years. Some risk factors that increase the estrogen level are: obesity, early menarche, late menopause, nulliparity and tamoxifen use. Women with Lynch syndrome are also at an elevated risk of endometrial cancer, along with other malignancies [2]. Most cases are diagnosed while in stage I, limited to the uterus. Fiveyear survival rate after surgery is very high, $88 \%$ [3].

Laparotomy (LPT) has always been considered, for many years, the standard approach for endometrial cancer surgical staging. Current trend is minimally invasive surgery. Many authors are publishing studies with excellent results using the laparoscopic approach [4-7]. Although some reviews of clinical trials have criticized the possibility of bias, like the use of different inclusion criteria, most of the reviews favor LPS over LPT in terms of: less postoperative complications, earlier return to work and better quality of life, while it does not seem to have a worst global survival $[8,9]$. The present study seeks to show the results on endometrial cancer treatment comparing LPS vs LPT, at a medium volume university hospital.

\section{MATERIALS AND METHODS}

We designed a prospective observational study between 
January 2007 and April 2012 at the Ciudad Real University General Hospital in Spain. Every patient diagnosed of endometrial cancer and subsidiary to surgical staging was included. Diagnosis confirmation was performed in every case with hysteroscopy and endometrial biopsy. Extension study prior to surgery was performed with physical examination, blood sampling and CA 125 determination, transvaginal and transabdominal sonography. Every patient was also studied with nuclear magnetic resonance and/or computed tomography. Patients in advanced stages, not subsidiary to surgery treatment were excluded.

Every patient agreed to participate and signed informed consent. Surgeries were performed at our center by the same team of gynecologic surgeons. Surgery protocol was performed independently of the approach: abdominal cavity exploration, peritoneal washing, total extrafascial hysterectomy, bilateral anexectomy and pelvic lymphadenectomy with or without paraaortic lymphadenectomy. When paraaortic lymphadenectomy was performed it reached the left renal vein. Apendicectomy and supra and infracolic radical omentectomy was performed in those patients with an unfavorable histology (clear cell carcinoma and papillary serous carcinoma).

Histological analysis was performed at our center. 1998 FIGO staging was used until the end of 2009, and then we used the new 2009 FIGO staging [10]. Adjuvant treatment, when needed, was also applied at our center. Follow-up visits were scheduled every 3 months for the first 2 years, every 6 months for 3 years, and yearly thereafter.

\section{Statistical Analysis}

Cualitative variables were defined using frequency distribution tables and sector graphics. Pearson $\chi^{2}$ test was used for statistical analysis. Cuantive variables were studied using central tendency statistics (mean, median and mode), dispersion statistics (standart deviation) and represented using histograms. Inferencial statistic: Kolmogórov-Smirnov test was used to evaluate normal distribution of the sample. Means comparison was performed using Student $\mathrm{T}$ and Mann-Whitney U Test for the not normally distributed variables.

Disease-free survival (DFS) was defined as the period (in months) from surgery until the date of first recurrence. Overall survival (OS) was calculated from the date of surgery to the date of death.

DFS and OS were estimated by Kaplan-Meier survival analysis, the log-rank test was used to compare both surgical approaches. A p value less than 0.05 was considered statistically significant. Statistical analysis was performed using the Statistical Package for Social Science for Windows (SPSS, Inc., Chicago, IL) version 19.0.

\section{RESULTS}

70 women diagnosed of endometrial cancer were included. 49 cases $(70 \%)$ were approached using LPS, while 21 cases $(30 \%)$ approached using LPT. Groups distribution is shown in Table 1. There was no statistical significant difference between the two groups in the general characteristics. $3(4.28 \%)$ intraoperative complications were reported, 2 of them in the LPS group: a vesical perforation resolved laparoscopically, and a case of hypercapnia secondary to pneumoperitoneum that requiered LPT conversion. In the LPT group a case of bleeding was reported, it required intraoperative blood transfusion. There were no statistical significant differences in surgery duration between both groups. LPS mean time was $183.06+-21.03$ minutes (range 120 230) and LPT mean time was $195.24+/-28.39$ minutes (range 130 - 240). Mean difference 12.16 (95\% CI - 0.2 24).

Peritoneal cytology was positive in 2 cases $(2.85 \%)$, one case in each group. Pelvic lymphadenectomy was performed in every patient of the study. 15 patients (21.4\%) also received paraaortic lymphadenectomy, 14 in the LPT group and 1 in the LPS group. There were no statistically significant differences in the number of pelvic nodes resected between both techniques $13.9 \pm 6.86$ (range 4 - 28) for LPS and $13.43 \pm 6.46$ (range 4 - 31) for LPT. The mean number of paraaortic nodes resected was $7.2 \pm 3.3$ (range 3 - 10). In 3 patients node metastasis was identified, all of them in the LPS group and affecting the pelvic region. Blood loss was significantly lower in the LPS group: $2.1 \mathrm{~g} / \mathrm{dl}$ haemoglobin decrement after the surgery vs $3.97 \mathrm{~g} / \mathrm{dl}$ in the LPT group $(\mathrm{p}<0.05) .6 .02 \%$ haematocrit decrement vs $9.47 \%(\mathrm{p}<0.05)$. Post-operative complications were also significantly lower in the LPS group: $3(6.12 \%)$ vs $6(28.5 \%)$ in the LPT group (p $<0.01$ ).

In the first group complications were: one cameratrocar wound seroma, vesico-vaginal fistula (resolved using an urine catheter for 1 month) and one case of strength loss in a myopathy affected patient. In the LPT group: 2

Table 1. Patient characteristics.

\begin{tabular}{|c|c|c|c|}
\hline Patient data & $\begin{array}{c}\text { Laparoscopy } \\
(\mathrm{N}=49) \\
\text { mean } \pm \mathrm{SD}(\text { range })\end{array}$ & $\begin{array}{c}\text { Laparotomy } \\
(\mathrm{N}=21) \\
\text { mean } \pm \mathrm{SD}(\text { range })\end{array}$ & $\mathrm{p}$ value \\
\hline Age (years) & $63 \pm 11(34-84)$ & $65 \pm 11(39-85)$ & NS \\
\hline BMI $\left(\mathrm{kg} / \mathrm{m}^{2}\right)$ & $32 \pm 9.5(19-51)$ & $27 \pm 4.8(19-36)$ & NS \\
\hline CA 125 (UI) & $13.2 \pm 2.32$ & $14.8 \pm 2.45$ & NS \\
\hline $\begin{array}{c}\text { Pre-operative } \\
\text { haemoglobin }(\mathrm{g} / \mathrm{dl})\end{array}$ & $13.8 \pm 1.02$ & $13.34 \pm 1.19$ & NS \\
\hline $\begin{array}{c}\text { Pre-operative } \\
\text { hematocrit (\%) }\end{array}$ & $39.14 \pm 7.97$ & $37.22 \pm 9.76$ & NS \\
\hline
\end{tabular}


abcesses, 2 cases of surgical incision infection and an intestinal subocclussion. None of them required invasive techniques or surgery for resolution. Hospital stay was significantly shorter in LPS patients: $4.29 \pm 1.62$ days vs $8.81 \pm 3.37$ days in the LPT group $(\mathrm{p}<0.01)$, mean difference: 4.52 (95\% IC 3.3 - 5.7). Histology distribution and final staging are reflected in Table 2.

30 of the 49 patients treated laparoscopically (61\%) received adjuvant therapy, 22 brachyteraphy, 3 brachyteraphy plus external radiotherapy and 5 radiotherapy plus chemotherapy. 14 of the 21 patients $(66 \%)$ in the LPT group received adjuvant therapy after the surgery, 5 brachitherapy, 4 brachiteraphy plus external radiotherapy and 5 radiotherapy plus chemotherapy. There was no statistically significant difference in the adjuvant treatment between both groups.

Mean follow-up was 23.41 months (range 6 - 53). The number of deaths did not differ significantly, $2(4 \%)$ in the LPS group and 3 (14\%) in the LPT group. Global survival was numerically but not statistically greater in the LPS approach: $92.79 \%$ were alive at the end of the follow-up vs $77.21 \%$ (log-rank test $\mathrm{p}=0.07)$ (Figure 1). Recurrences were more frequent in the LPT group 5 $(23 \%)$ vs $2(4 \%)(p=0.01)$. Disease-free survival is reflected in Figure 2. Survival curves were more homogenous and no significant difference was found when only the patients with stage I endometrial cancer were compared $(\log$-rank test $p=0.051)$ Figure 3 .

\section{DISCUSSION}

Our results offer no surprises and agree with most of the recently published studies. There were no differences in intraoperative complications. There were no vascular complications during lymphadenectomy. Laparotomy conversion was scarce ( 1 case, $2 \%$ ). Disagreeing with the Palomba et al. metaanalysis [11] where four trials were pooled and analysed who found LPS duration significantly longer than LPT in our study surgery duration was similar between both approaches. Malzoni et al. [12] prospective study performed lymphadenectomy in every

Table 2. Final staging and histology.

\begin{tabular}{ccccc}
\hline Stage & Laparoscopy $(\mathrm{N}=49)$ & \multicolumn{2}{l}{ Laparotomy $(\mathrm{N}=21)$} \\
\hline I & 42 & $85.70 \%$ & 15 & $71.4 \%$ \\
II & 2 & $4.10 \%$ & 4 & $19 \%$ \\
III & 5 & $10.30 \%$ & 2 & $9.6 \%$ \\
Histology & & & & \\
Endometrioid & 48 & $97.90 \%$ & 15 & $71.4 \%$ \\
Papillary serous & 1 & $2.10 \%$ & 3 & $14.3 \%$ \\
Clear cell & 0 & $0 \%$ & 3 & $14.3 \%$ \\
\hline
\end{tabular}

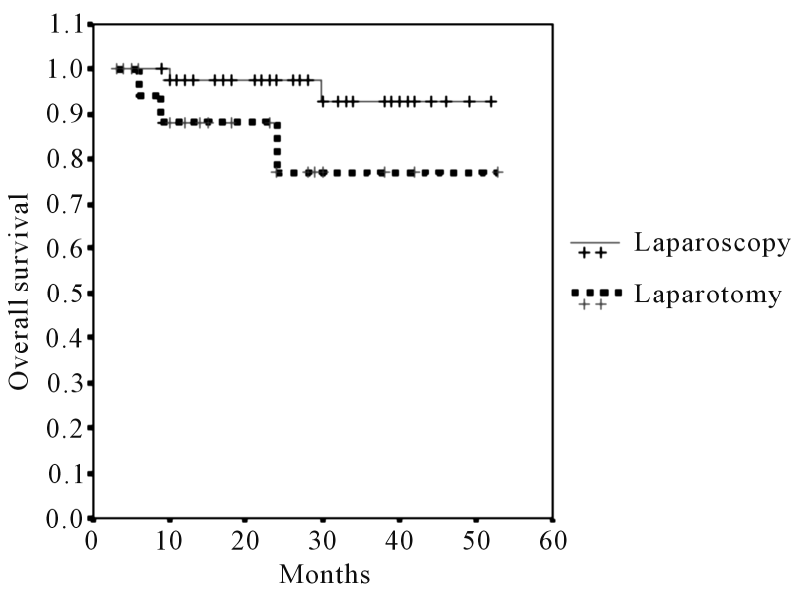

Figure 1. Kaplan-Meier overall survival estimates.

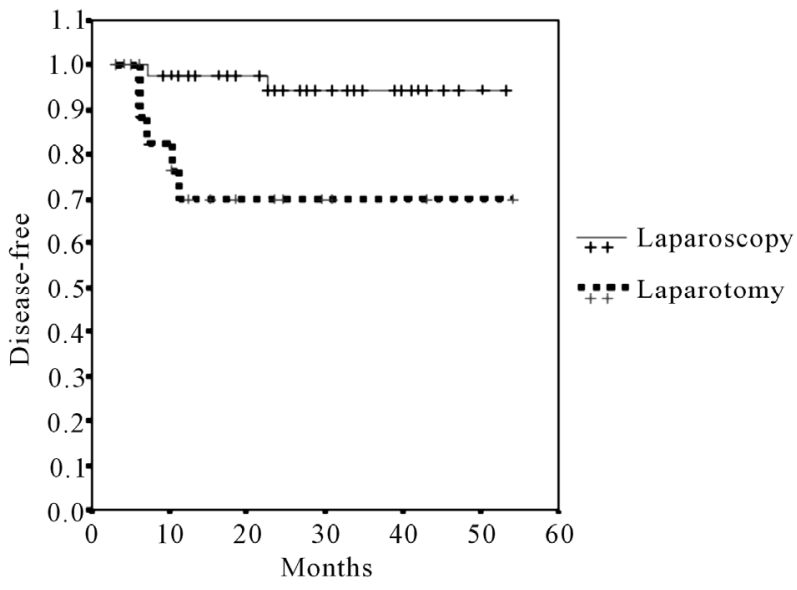

Figure 2. Kaplan-Meier disease-free survival estimates.

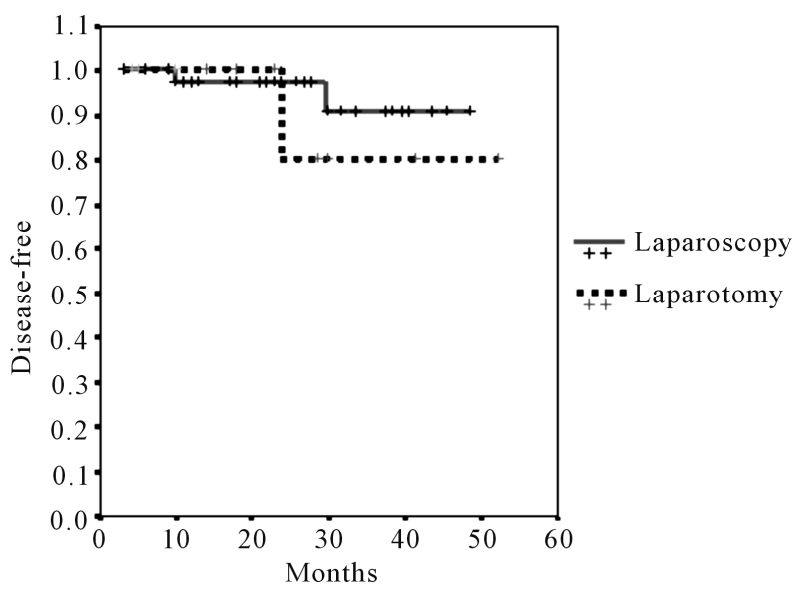

Figure 3. Stage I carcinoma. Kaplan-Meier overall survival estimates.

patient, also found a longer surgery time in the LPS group. In our study the mean LPS surgery time is slightly longer than published by other authors. A possible reason might be the beginning of the learning curve, responsible for longer surgeries during the first year of study. During 
the last year of our study mean LPS duration was around 140 minutes. According to our bleeding estimation, LPS has the advantage of significant decrement in blood loss compared to the LPT. Coronado et al. [13] recently published study showed similar results. One of the advantages of the LPS is image magnification that allows a safer vascular dissection during the lymphadenectomy. Pneumoperitoneum helps in the dissection of avascular spaces. The new vascular closure devices also help to minimize the blood loss.

The mean number of lymph nodes resected with LPS is 13.9. In our study no significant difference was found between groups. This finding is similar to the majority of the published studies [12-17]. In Zullo et al. [9] metaanalysis the mean number of nodes obtained ranged from 8.86 to 23.5 . We decided to perform pelvic lymphadenectomy to every patient because of its low morbidity. Paraaortic lymphadenectomy was added in those cases of unfavorable histology or in the presence of recurrence risk factors.

In our study, postoperative complications were significantly lower in the LPS group. Only 3 cases, all of them mild, resolved without reintervention. Recently published studies show the same results. Zhang et al. [18] recent revision includes 8 randomized controlled trials (RCT) and 3599 patients, also showed a lower number of postoperative complications in the LPS group (OR 0.59. $95 \%$ CI $0.46-0.75 ; \mathrm{p}<0.001)$. Globally, the decreased blood loss and a lower number of complications make possible a shorter hospital stay, an earlier incorporation to daily life and a better quality of life. In a public health system like ours, this also means an important way of saving money.

In our study the LPS group mean hospital stay was half of that with the LPT. In the multicenter LACE study [5] the quality of life level was compared in 322 women, a significantly higher score was found in the LPS group at first, third and sixth months. Zullo et al. [17] Italian group obtained similar results. On the other hand, the GOG LAP-2 [4] trial found an initial better quality of life but no difference after the first 6 months. Our study did not show significant difference in overall survival, nonetheless recurrence was significantly more frequent in the LPT group.

We know that a selection bias might exist, as the LPT group had more cases of unfavorable histology (clear cell carcinoma and papillary serous carcinoma), while the LPS group histology was mostly endometrioid. This might have caused a shorter disease free time in the LPT group, although OS has not been significantly affected. We compared only the patients in stage I, trying to homogenize the data and avoid bias. This way we found no statistical difference in OS. Most of the RCTs [12,16,17] show the same results, although there are not many stud- ies that evaluate survival and even less for a long period (Tozzi [16] 44 months, Zullo [17] 79 months, Malzoni [12] 38.5 months). Older studies neither show differences in OS: Malur [19] found no differences studyingin 57 patients, Pignotti [20] with a 45 months follow-up obtained 5.3\% recurrences in the LPS group and $7.4 \%$ in the LPT group. In our study mean follow-up was 23 months with a maximum of 56 months. Palomba [11] meta-analysis emphasises the need of longer RCTs to obtain concluyent results.

It is important to mention than most of the published studies include initial stage endometrial cancers with a high grade of differentiation (type I) with good prognosis and high 5-year survival. The question is if the minimal invasive approach would be as safe as the laparotomy when applied in patients with more unfavorable histologies like the clear cell carcinoma, the papillary serous carcinoma or poorly differentiated endometroid carcinoma (type II). Feder et al. [21] recent study includes 383 high grade cases, treated at a high volume oncology center, it showed favorable results towards the LPS, smaller number of complications, shorter hospital stay and the same OS as the LPT.

Finally, our study has been performed at a university hospital with a medium volume of oncology surgery. According to our results we believe that minimally invasive surgery approach for endometrial cancer is possible in this kind of center. Beverly et al. [22] published a retrospective study with 86 patients (50 LPT and 36 LPS) treated at a low volume military hospital, it showed the same results that a high volume center: LPS achieved lower blood loss and the same rate of complications and lymphatic nodes as the LPT.

They also mention that the hospital stay and the cost per procedure are even lower than those reported in the high-volume centers [23].

\section{CONCLUSION}

The LPS approach for the endometrial cancer offers multiple advantages over the LPT such as a lower rate of complications, minor blood loss, and a shorter hospital stay while offering the same oncological radicality and OS as the LPT.

More RCTs, with a long follow up period, are needed to strengthen these results.

\section{REFERENCES}

[1] Siegel, R., Naishadham, D. and Jemal, A. (2012) Cancer statistics, 2012. CA: A Cancer Journal for Clinicians, 62, 10-29. doi: $10.3322 /$ caac. 20138

[2] Van den Bosch, T., Coosemans, A., Morina, M., et al. (2012) Screening for uterine tumours. Best Practice \& Research Clinical Obstetrics \& Gynaecology, 26, 257 - 
266. doi:10.1016/j.bpobgyn.2011.08.002

[3] Creasman, W.T., Odicino, F., Maisonneuve, P., et al. (2006) Carcinoma of the corpus uteri. FIGO 26th annual report on the results of treatment in gynecological cancer. International Journal of Gynecology \& Obstetrics, 95, S105-S143. doi:10.1016/S0020-7292(06)60031-3

[4] Kornblith, A.B., Huang, H.Q., Walker, J.L., et al. (2009) Quality of life of patients with endometrial cancer undergoing laparoscopic international federation of gynecology and obstetrics staging compared with laparotomy: A gynecologic oncology group study. Journal of Clinical Oncology, 27, 5337-5342. doi:10.1200/JCO.2009.22.3529

[5] Janda, M., Gebski, V., Brand, A., et al. (2010) Quality of life after total laparoscopic hysterectomy versus total abdominal hysterectomy for stage I endometrial cancer (LACE): A randomised trial. The Lancet Oncology, 11, 772-780. doi:10.1016/S1470-2045(10)70145-5

[6] Mourits, M.J., Bijen, C.B., Art, H.J., et al. (2010) Safety of laparoscopy versus laparotomy in early-stage endometrial cancer: A randomised trial. The Lancet Oncology, 11, 763-771. doi:10.1016/S1470-2045(10)70143-1

[7] Walker, J.L., Piedmonte, M.R., Spirtos, N.M., et al. (2009) Laparoscopy compared with laparotomy for comprehensive surgical staging of uterine cancer: Gynecologic oncology group study LAP2. Journal of Clinical Oncology, 27, 5331-5336. doi:10.1200/JCO.2009.22.3248

[8] Carter, J.R. (2011) Laparoscopy or laparotomy for endometrial cancer? A review of three prospective randomised trials. Australian and New Zealand Journal of Obstetrics and Gynaecology, 51, 387-392. doi:10.1111/j.1479-828X.2011.01306.x

[9] Zullo, F., Falbo, A. and Palomba, S. (2012) Safety of laparoscopy vs laparotomy in the surgical staging of endometrial cancer: A systematic review and metaanalysis of randomized controlled trials. American Journal of $\mathrm{Ob}$ stetrics and Gynaecology, 13, 94-100. doi:10.1016/j.ajog.2012.01.010

[10] Pecorelli, S. (2009) Revised FIGO staging for carcinoma of de vulva, cervix and endometrium. International Journal of Obstetrics and Gynaecology, 105, 103-104. doi:10.1016/j.ijgo.2009.02.012

[11] Palomba, S., Falbo, A., Mocciaro, R., et al. (2009) Laparoscopic treatment for endometrial cancer: A meta-analysis of randomized controlled trials (RCTs). Gynecologic Oncology, 112, 415-421. doi:10.1016/j.ygyno.2008.09.014

[12] Malzoni, M., Tinelli, R., Cosentino, F., et al. (2009) Total laparoscopic hysterectomy versus abdominal hysterictomy with lymphadenectomy for early-stage endometrial cancer: A prospective randomized study. Gynecologic Oncology, 112, 126-133. doi:10.1016/j.ygyno.2008.08.019

[13] Coronado, P.J., Herraiz, M.A., Magrina, J.F., et al. (2012)
Comparison of perioperative outcomes and cost of robotic-assisted laparoscopy, laparoscopy and laparotomy for endometrial cancer. European Journal of Obstetrics \& Gynecology and Reproductive Biology, 165, 289-294. doi:10.1016/j.ejogrb.2012.07.006

[14] Fram, K.M. (2002) Laparoscopically assisted vaginal hysterectomy versus abdominal hysterectomyin stage I endometrial cancer. International Journal of Gynecological Cancer, 12, 57-61. doi:10.1046/j.1525-1438.2002.01038.x

[15] Zorlu, C.G., Simsek, T. and Ari, E.S. (2005) Laparoscopy or laparotomy for the management of endometrial cancer. Journal of the Society of Laparoendoscopic Surgeons, $\mathbf{9}$, 442-446.

[16] Tozzi, R., Malur, S., Koehler, C., et al. (2005) Laparoscopy versus laparotomy in endometrial cancer: First analysis of survival of a randomized prospective study. Journal of Minimally Invasive Gynecology, 12, 130-136. doi:10.1016/j.jmig.2005.01.021

[17] Zullo, F., Palomba, S., Russo, T., et al. (2005) A prospective randomized comparison between laparoscopic and laparotomic approaches in women with early stage endometrial cancer: A focus on the quality of life. American Journal of Obstetrics \& Gynecology, 193, 1344-1352. doi:10.1016/j.ajog.2005.02.131

[18] Zhang, H., Cui, J., Jia, L., et al. (2012) Comparison of laparoscopy and laparotomy for endometrial cancer. International Journal of Obstetrics \& Gynecology, 116, 185-191. doi:10.1016/j.ijgo.2011.10.022

[19] Malur, S., Possover, M., Michels, W., et al. (2001) Laparoscopic-assisted vaginal versus abdominal surgery in patients with endometrial cancer a prospective randomized trial. Obstetrics \& Gynecology, 80, 239-244. doi:10.1006/gyno.2000.6069

[20] Pignotti, E., De Aloysio, D. and De Iaco, P. (2005) Is total laparoscopic surgery for endometrial carcinoma at risk of local recurrence? A long-term survival. Anticancer Research, 25, 2423-2428.

[21] Fader, A., Seamon, L., Escobar, P., et al. (2012) Minimally invasive surgery versus laparotomy in women with high grade endometrial cancer: A multi-site study performed at high volume cancer centers. Obstetrics \& Gynecology, 126, 180-185. doi:10.1016/j.ygyno.2012.04.028

[22] Reed, B.G., Lowery, W.J., Keyser, E.A., et al. (2011) Surgically managed stage I endometrial cancer in a lowvolume center: Outcomes and complications in a military residency program. American Journal of Obstetrics \& Gynecology, 205, e1-e5.

[23] Diaz-Montes, T.P., Zahurak, M.L., Giuntoli, R.L., et al. (2007) Concentration of uterine cancer surgical care among the elderly: A population-based perspective. Gynecologic Oncology, 107, 436-440. doi:10.1016/j.ygyno.2007.07.060 